\title{
A FRAMEWORK FOR MRI IMAGE RETRIEVAL USING CURVELET TRANSFORM AND EUCLIDEAN DISTANCE
}

\author{
Rajakumar and Muttan \\ Department of Electronics and Communication Engineering, Guindy, Anna University, Chennai, India
}

Received 2013-01-21, Revised 2013-03-10; Accepted 2013-04-12

\begin{abstract}
Content Based Image Retrieval (CBIR) system supports users to retrieve relevant medical images based on their features. Current content based image retrieval systems are incapable of providing exact results to the users. To address this problem a consistent feature extraction method is required for content based medical image retrieval system to extract similar features from the images. In this study discrete curvelet based feature extraction technique is proposed to retrieve similar images and the similarity distance is calculated by using Euclidean distance. The proposed method gives better precision and recall rate. Experimental results on a database of 200 MRI images.
\end{abstract}

Keywords: Content Based Image Retrieval, Texture Retrieval, Discrete Curvelet, Similarity Matching, Euclidean Distance

\section{INTRODUCTION}

In current years, fast advances in technology have created a huge amount of image data in different areas, such as fine art galleries, fashion design, medicine. We often need to efficiently retrieve image data to perform assigned tasks and to make a decision. Hence, developing proper tools for the retrieval image from huge image collections is difficult. Two different types of approaches, i.e., text based image retrieval and content based image retrieval. In the text-based image retrieval system, the images are physically annotated by text descriptors and then used by a database system to perform image retrieval. Though, there are some restrictions of using keywords to attain image retrieval. That is, the textual descriptions given by an annotator could be different from the perspective of a user. In further, there are inconsistencies between user textual queries and image annotations. To alleviate these problems, the image retrieval is carried out according to the contents of image.

Such approach is the so-called Content-Based Image Retrieval (CBIR). The main aim of the CBIR system is to retrieve the efficient images. The feature extraction in CBIR is a major step whose efficiency depends upon the technique adopted for extracting features from particular images. The CBIR utilizes visual contents of an image such as color, texture, shape, spatial layout, to represent and index the image database. Along with these features, texture feature is a more important and major visual property of an image. A comprehensive and extensive literature survey on CBIR is presented in (Zhang et al., 2012; Zajic et al., 2011). At present, there are many techniques for capturing medical images from patients to assist with diagnostic tasks. These include Computed Tomography (CT), Magnetic Resonance Imaging (MRI), X-ray images, ultrasonography. The diagnostic job generates a huge amount of medical images that must be archived for upcoming evaluations. The majority of these techniques produce digital images, which are archived and handled through Picture Archiving and Communication Systems (PACS). Content based image retrieval is more challenging in medical domain than in many general purpose domains. The main reason is that important features in medical Corresponding Author: Rajakumar, Department of Electronics and Communication Engineering, College of Engineering, Guindy, Anna University, Chennai, India 
images are often local features rather than global features. This makes feature extraction much more demanding. Feature extracted for medical images must be able to both describe fine details of the images and allow quick retrieval of relevant images. The rest of this study is organized as follows. The background work is presented in Section 2. The discrete curvelet transform is described in Section 3. Curvelet based feature extraction approach is discussed at section 4. The experimental results and conclusion are reported in Section 5 and 6.

\subsection{Related Works}

In general the spectral approaches of texture feature representation, wavelet transforms and Gabor filters have been found to be used in CBIR successfully. The multiresolution wavelet transform has been employed to retrieve images. The wavelet features do not achieve high level of retrieval accuracy. Therefore, different approaches have been developed to achieve higher level of retrieval accuracy using wavelet transform. Wavelets perform better for one dimensional signal as it is good in representing point discontinuities. All singularities in a one dimensional signal are point singularities, so wavelets have certain universality there. However, in higher dimensions, more types of singularities may exist. In these cases wavelets lose their universality (Quellec et al., 2012; Zhang et al., 2012; Zajic et al., 2011) and perform merely good enough in capturing the edge discontinuities in 2-D space, which is important in texture representation. Another multiresolution approach, the Gabor filters, consists of a group of wavelets each of which capturing energy at a specific resolution and orientation. To overcome the problems in using the discrete wavelet and Gabor filters transform, a new multiresolution approach named discrete curvelet transform. Curvelets take the form of basis elements, which have elongated effective support; i.e., length > width (Akakin and Gurcan, 2012). Therefore, curvelets can capture anisotropic elements such as the edges of an image effectively (Li et al., 2011).

Furthermore, curvelet spectra cover the frequency plane of an image completely. For these important properties, curvelet transform can be used as a powerful image feature capturing tool in CBIR. Texture feature representation and its use in CBIR is an important research issue. Though many works on texture classification and representation have already been done, it is still an open issue. In order to realize the effectiveness of the curvelet features for CBIR, we make a systematic analysis, application and evaluation of this feature in this study.

\subsection{Discrete Curvelet Transform}

Fundamentally, curvelet transform broaden the ridgelet transform to multiple scale analysis. Given an image $f(x, y)$, the continuous ridgelet coefficients are expressed in Equation (1) as shown below:

$$
\mathrm{R}_{\mathrm{f}}(\mathrm{a}, \mathrm{b} . \theta)=\iint \psi_{\mathrm{a}, \mathrm{b} . \theta}(\mathrm{x}, \mathrm{y}) \mathrm{f}(\mathrm{x}, \mathrm{y}) \mathrm{dxdy}
$$

Here, $a$ is the scale parameter where $a>0, b \in R$ is the translation parameter and $\theta \in[0,2 \pi)$ is the orientation parameter. Exact reconstruction is possible from these coefficients. A ridgelet can be defined in Equation (2):

$\psi_{\mathrm{a}, \mathrm{b}, \theta}(\mathrm{x}, \mathrm{y})=\mathrm{a}^{\frac{1}{2}} \psi\left(\frac{\mathrm{x} \cos \theta+\mathrm{y} \sin \theta-\mathrm{b}}{\mathrm{a}}\right)$

where, $\theta$ is the orientation of the ridgelet. Ridgelets are constant along the lines $\mathrm{x} \cos \theta+\mathrm{y} \sin \theta=$ const and transverse to these ridges are wavelets (Zhang et al., 2012; Zajic et al., 2011). Ridgelets take the form of a basis element and obtain a high anisotropy. Therefore, it captures the edge information more effectively. A ridgelet is linear in its edge direction and is much sharper than a conventional sinusoidal wavelet (Zhang et al., 2012; Zajic et al., 2011). Curvelet transform based on wrapping of Fourier samples takes a 2-D image as input in the form of a Cartesian array $\mathrm{f}[\mathrm{m}, \mathrm{n}]$ such that $0 \leq \mathrm{m}<\mathrm{M}, 0 \leq \mathrm{n}<\mathrm{N}$ and generates a number of curvelet coefficients indexed by a scale $\mathrm{j}$, an orientation 1 and two spatial location parameters $\left(k_{1}, k_{2}\right)$ as output. To form the curvelet texture descriptor, statistical operations are applied to these coefficients. Discrete curvelet coefficients can be defined by Equation (3) (Liu et al., 2011; Manipoonchelvi and Muneeswaran, 2011):

$$
C^{D}\left(j, 1, k_{1}, k_{2}\right)=\sum_{0 \leq m \leq M} f[m, n] \phi_{j, 1, k_{1}, k_{2}}^{D}[m, n]
$$

Here, each, $\phi^{\mathrm{D}}{ }_{\mathrm{j}, 1, \mathrm{k}_{1}, \mathrm{k}_{2}}[\mathrm{~m}, \mathrm{n}]$ is a digital curvelet waveform. This curvelet approach implements the effective parabolic scaling law on the subbands in the frequency domain to capture curved edges within an image more effectively. Curvelets exhibit an oscillating behavior in the direction perpendicular to their orientation in frequency domain. Basically, wrapping 
based curvelet transform is a multiscale transform with a pyramid structure consisting of many orientations at each scale. At high scales, the curvelet waveform becomes so fine that it looks like a needle shaped element. To achieve higher level of efficiency, curvelet transform is usually implemented in the frequency domain. That is, both the curvelet and the image are transformed and are then multiplied in the Fourier frequency domain. The product is then inverse Fourier transformed to obtain the curvelet coefficients. The process can be described as Curvelet transform = IFFT[FFT(Curvelet) FFT(Image)] and the product from the multiplication is a wedge.

\subsection{Curvelet based Feature Extraction}

A generalized form of content based image retrieval process using curvelet transform is shown in Fig. 1. In our retrieval experiments, texture features are first computed from the curvelet coefficients of the query image. The database images are then compared to the query image using a similarity measurement technique on the feature elements in the features vectors. The system uses the Euclidean distance to compare the query feature vector with all the feature vectors in the feature database. Figure 2 describes the flowgraph of curvelet transform.

\subsection{Similarity Measures}

The similarity measure by a given query image involves searching the database for similar curvelet coefficients. Euclidean Distance is suitable and effective method which is widely used in image retrieval area. The retrieval results are a list of medical images ranked by their similarities measure with the query image (Wang and Zhao, 2010; Prasad and Krishna, 2011). The similarity distance measure between the vectors of query image and the database image can be defined below as Equation 4 . where, $d$ is the distance between the feature vector and $n$ represents the total number of pixels in the image.

The Euclidean distance ' $d$ ' can be expressed in Equation (4):

$\mathrm{d}(\mathrm{Q}, \mathrm{T})=\left(\sum_{\mathrm{i}=1}^{2 \mathrm{n}}\left(\mathrm{Q}_{\mathrm{i}}-\mathrm{T}_{\mathrm{i}}\right)^{2}\right)^{\frac{1}{2}}$

The images in the database are ranked according to their distance $d$ to the query image in ascending orders and then the ranked images are retrieved. The computed distance is ranked according to closest similar; in addition, if the distance is less than a certain threshold set, the corresponding original images is close or match the query image. Precision $\mathrm{P}$ is defined as the ratio of the number of retrieved relevant images $r$ to the total number of retrieved images $n$, i.e., $P=r / n$ (Iakovidis et al., 2009). The Precision and Recall definition is given by Equation (5) and (6).

Precision measures the accuracy of the retrieval:

Precision $=\frac{\text { No. of relevant images retrieved }}{\text { Total no. of images retrieved }}=\frac{r}{n}$

Recall is defined by $\mathrm{R}$ and is defined as the ratio of the number of retrieved relevant images $r$ to the total number $\mathrm{m}$ of relevant images in the whole database, i.e., $\mathrm{R}=\mathrm{r} / \mathrm{m}$ (Iakovidis et al., 2009). Recall measures the robustness of the retrieval:

$$
\text { Recall }=\frac{\text { No. of relevant images retrieved }}{\text { Total no. of relevant images in DB }}=\frac{\mathrm{r}}{\mathrm{m}}
$$

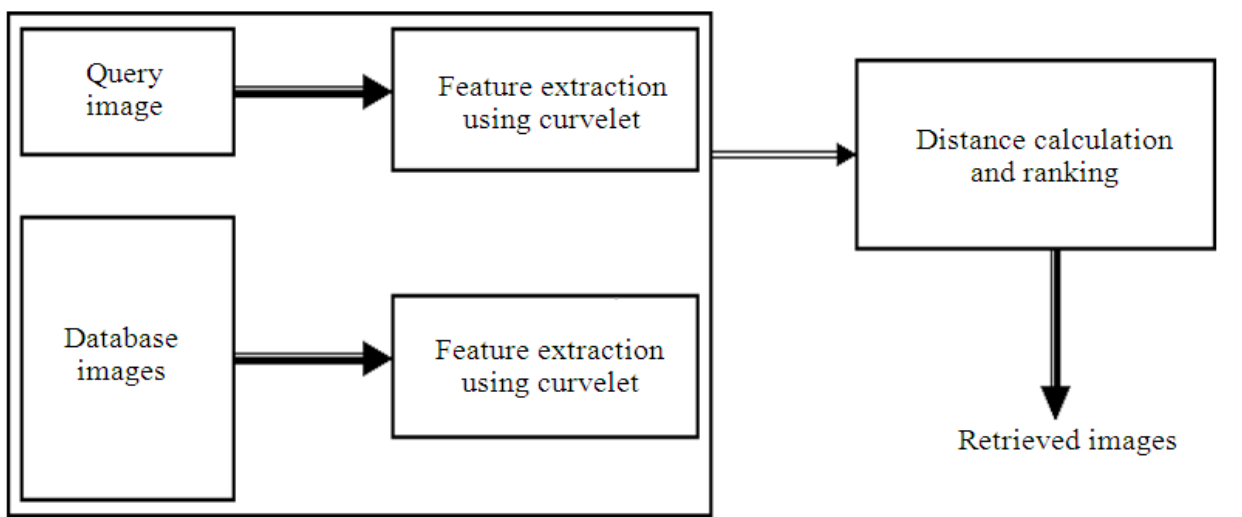

Fig. 1. Image retrieval mechanism using curvelet transform 
Rajakumar and Muttan / Journal of Computer Science 9 (3): 285-290, 2013

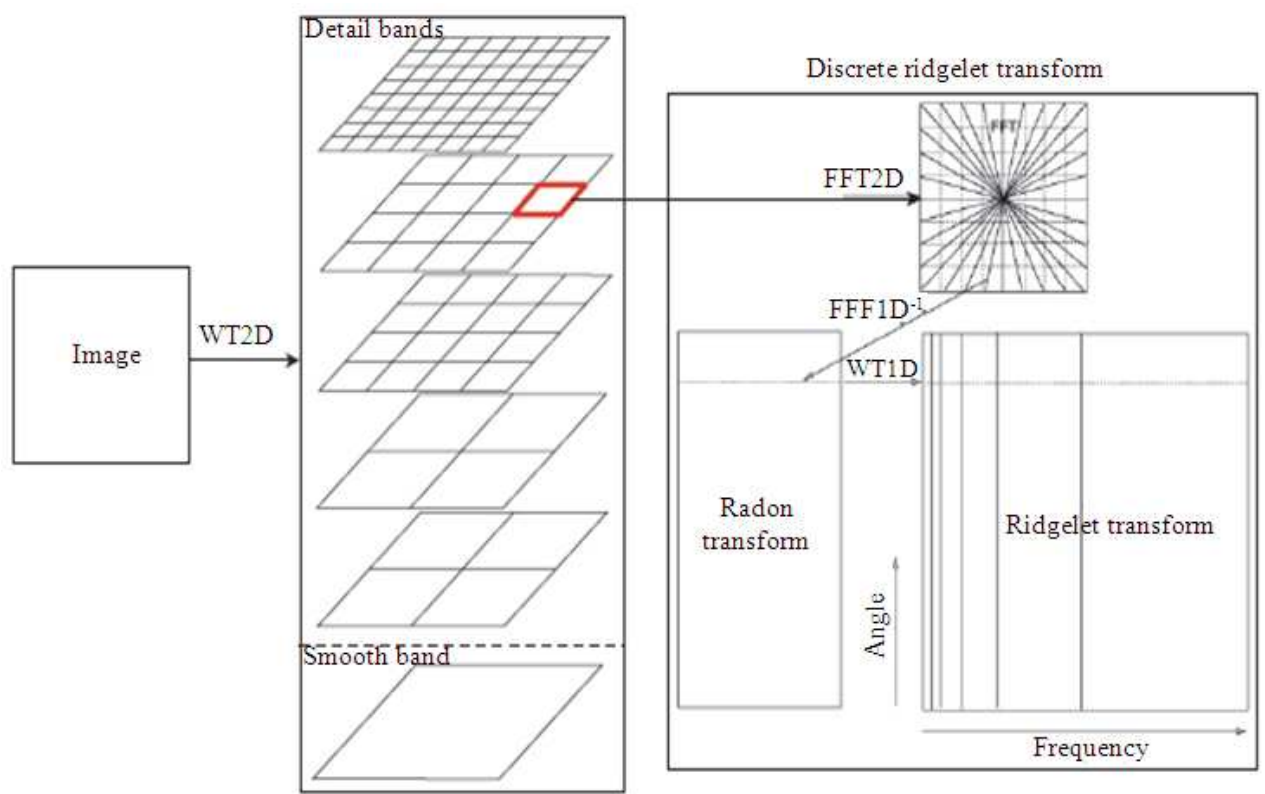

Fig. 2. Curvelet transform flow graph

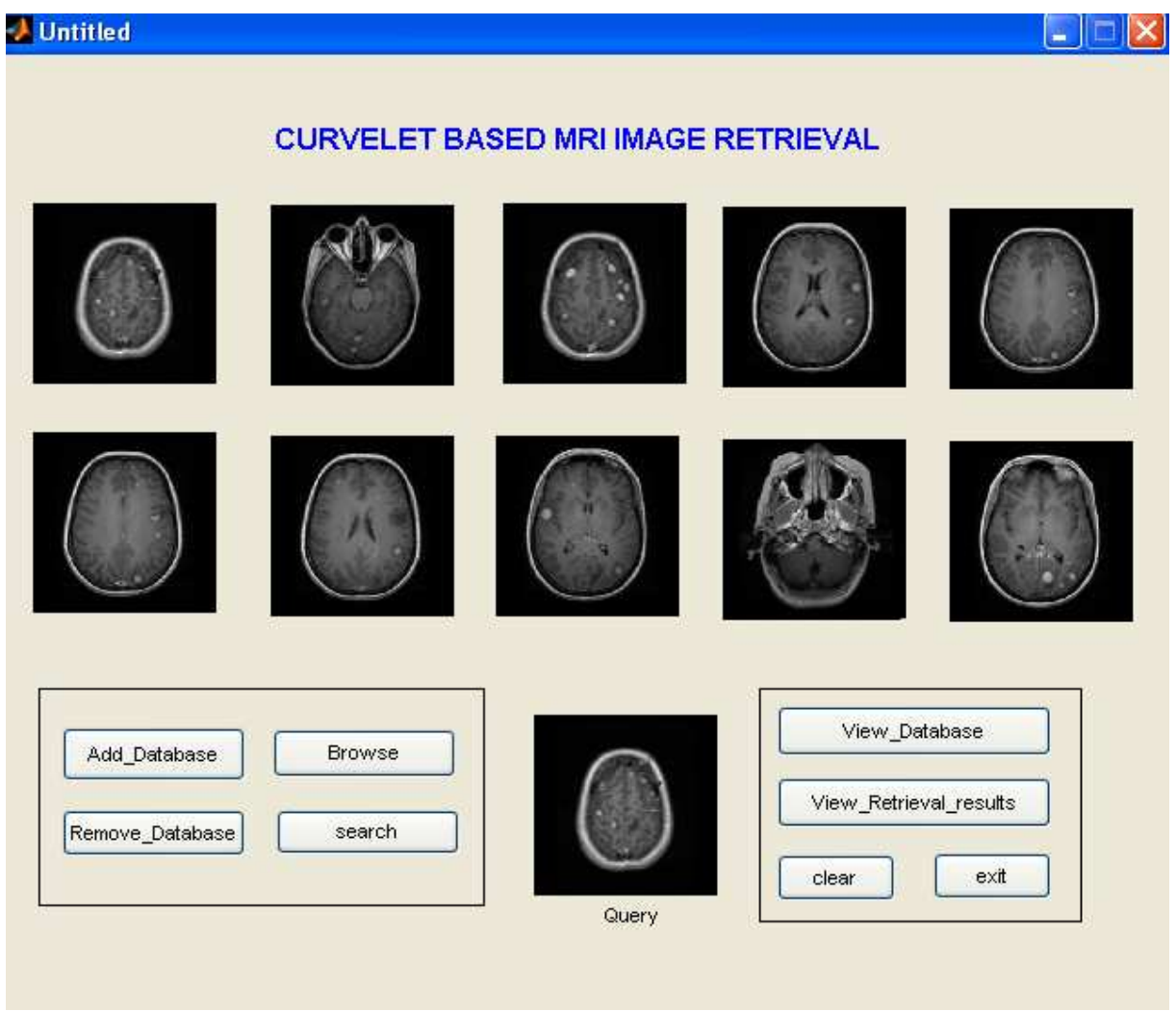

Fig. 3. GUI for Curvelet based MRI image retrieval 


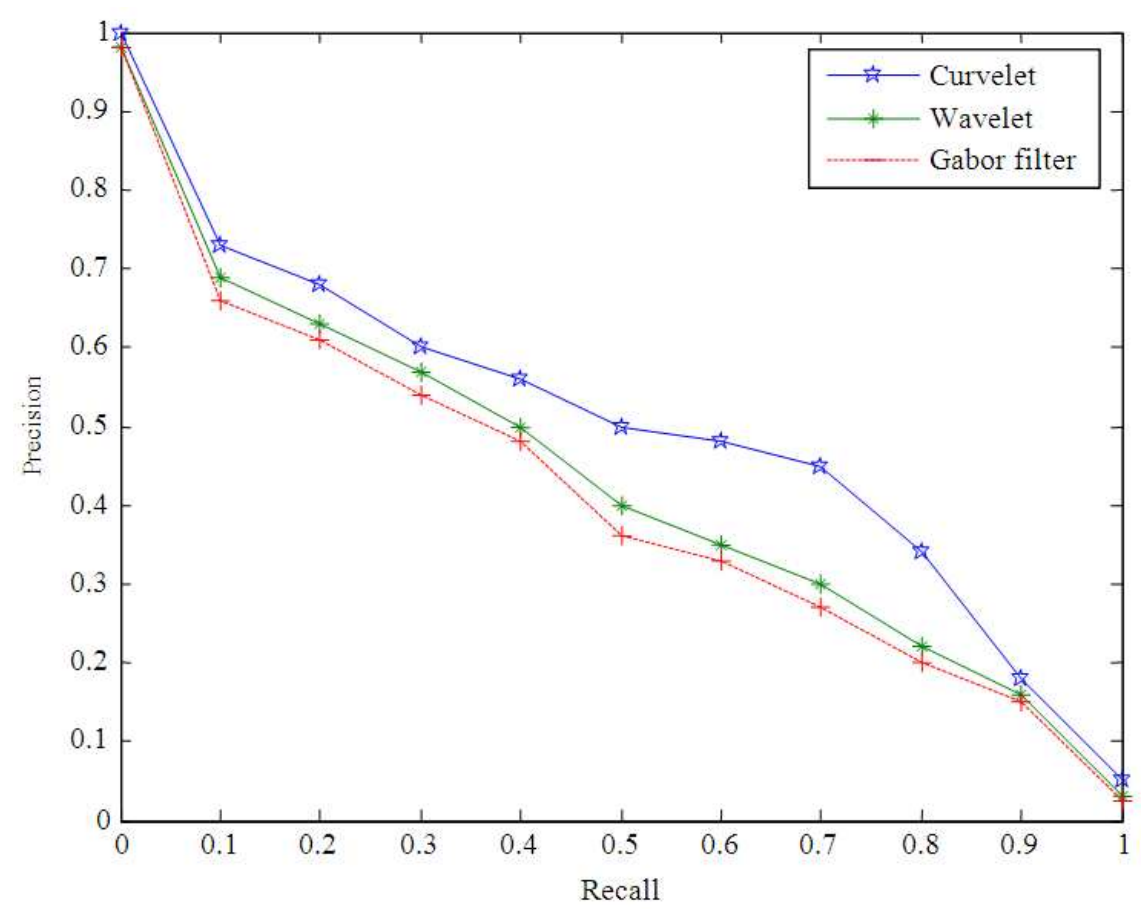

Fig. 4. Comparison of recall vs precision rate

Table 1. Various approaches and their retrieval time, accuracy

\begin{tabular}{lll}
\hline Approach & Total retrieval time for & $\begin{array}{l}\text { Average retrieval } \\
\text { accuracy }(\%)\end{array}$ \\
\hline Curvelet & 200 images (in seconds) & 84.17 \\
Wavelet & 29.09 & 76.69 \\
Gabor Filter & 32.91 & 73.50 \\
\hline
\end{tabular}

\subsection{Experimental Results}

We present the performance of MRI image retrieval using discrete curvelet transform and euclidean distance method. First, we present the results of image retrieval using the curvelet technique on a data set to evaluate its robustness and accuracy. Then, we present the comparison of curvelet with two different formulations. Here GUI is created to analyze the impact of the proposed method as shown in Fig. 3. Similarity measures like, Recall and precision is calculated for proposed algorithm and compared with other methods depicted in Fig. 4. Table 1 describes various approaches and their retrieval time, accuracy.

\section{CONCLUSION}

We have proposed a framework for MRI image retrieval using discrete curvelet transform and euclidean distance. The main objective of this study is to investigate and evaluate an effective and robust approach for texture representation and to use it in image retrieval. For this purpose, we have investigated the texture analysis using several approaches. Throughout the study characteristics of the main MRI methods, i.e., discrete wavelet and discrete curvelet were discussed. Finally, we compared the curvelet CBIR performance with that of the existing Gabor filters and wavelet. This is the first systematic evaluation of the image retrieval using curvelet transform. This work has found that curvelet features outperformed the existing texture features in both accuracy and efficiency. Furthermore, curvelet texture features can also be used combined with color features to retrieve color images. Combining discrete curvelet texture features with color features may improve the retrieval outcome. 


\section{REFERENCES}

Akakin, H.C. and M.N. Gurcan, 2012. Content-based microscopic image retrieval system for multi-image queries. IEEE Trans. Inform. Technol. Biomed., 16: 758-769. PMID: 22311866

Iakovidis, D.K., N. Pelekis, E.E. Kotsifakos, I. Kopanakis and H. Karanikas et al., 2009. A pattern similarity scheme for medical imageretrieval. IEEE Trans. Inform. Technol. Biomed., 13: 442-450. DOI: 10.1109/TITB.2008.923144

Li, Y., H. Gong, D. Feng and Y. Zhang, 2011. An adaptive method of speckle reduction and feature enhancement for sar images based on curvelet transform and particle swarm optimization. IEEE Trans. Geosci. Remote Sens., 49: 3105-3116. DOI: 10.1109/TGRS.2011.2121072

Liu, S., W. Cai, L. Wen, S. Eberl and M.J. Fulham et al., 2011. Localized functional neuroimaging retrieval using $3 \mathrm{D}$ discrete curvelet transform. Proceedings of the IEEE International Symposium on Biomedical Imaging: From Nano to Macro, Mar. 30-Apr. 2, IEEE Xplore Press, Chicago, IL., pp: 1877-1880. DOI: 10.1109/ISBI.2011.5872774

Manipoonchelvi, P. and K. Muneeswaran, 2011. Significant region based image retrieval using curvelet transform. Proceedings of the International Conference on Recent Advancements in Electrical, Electronics and Control Engineering, Dec. 15-17, IEEE Xplore Press, Sivakasi, pp: 291-294. DOI: 10.1109/ICONRAEeCE.2011.6129812
Prasad, B.G. and A.N. Krishna, 2011. Statistical texture feature-based retrieval and performance evaluation of CT brain images. Proceedings of the 3rd International Conference on Electronics Computer Technology, Apr. 8-10, IEEE Xplore Press, Kanyakumari, pp: 289-293. DOI: 10.1109/ICECTECH.2011.5941703

Quellec, G., M. Lamard, G. Cazuguel, B. Cochener and C. Roux, 2012. Fast wavelet-based image characterization for highly adaptive image retrieval. IEEE Trans. Image Process., 21: 1613-1623. DOI: 10.1109/TIP.2011.2180915

Wang, J. and X. Zhao, 2010. A new approach for image retrieval with integrated Euclidean distance and rotational correlation. Proceedings of the IEEE International Conference on Wireless Communications, Networking and Information Security, Jun. 25-27, IEEE Xplore Press, Beijing, China, pp: 391-395. DOI: 10.1109/WCINS.2010.5541806

Zajic, G., N. Kojic and B. Reljin, 2011. Searching image database based on content. Proceedings of the 19th Telecommunications Forum, Nov. 22-24, IEEE Xplore Press, Belgrade, pp: 1203-1206. DOI: 10.1109/TELFOR.2011.6143766

Zhang, L., L. Wang and W. Lin, 2012. Generalized biased discriminant analysis for content-based image retrieval. IEEE Trans. Syst. Man Cybern. B Cybern., 42: 282-90. PMID: 21968743 